\title{
Dielectric, piezoelectric, and pyroelectric anisotropy in KCl-modified grain-oriented bismuth vanadate ceramics
}

\author{
K. Shantha and K. B. R. Varma ${ }^{a}$ \\ Materials Research Centre, Indian Institute of Science, Bangalore-560 012, India
}

(Received 20 October 1997; accepted 8 June 1998)

\begin{abstract}
The effect of the additive $\mathrm{KCl}$, on the structural, microstructural, and polar properties of bismuth vanadate $(\mathrm{BiV})$ ceramics is investigated. The scanning electron microscopic (SEM) studies reveal a remarkable modification in the microstructure and the occurrence of high grain-orientation (75\%) on $\mathrm{KCl}$ addition. The energy dispersive $\mathrm{x}$-ray (EDX) analyses indicate the presence of chemically inhomogeneous distribution of $\mathrm{KCl}$, with core-shell-like grain structure. The $\mathrm{KCl}$-modified $\mathrm{BiV}$ samples exhibit a broad and depressed phase transition, with no frequency dispersion, as a result of the increased internal stress and the formation of core-shell-like grain structure. Significant anisotropies are observed in the dielectric, piezoelectric, and pyroelectric responses of these grain-oriented ceramic samples. These samples are characterized by near rectangular ferroelectric hysteresis loops, with a significant anisotropy in the $P_{r}\left(P_{r \perp} / P_{r \|}=2.43\right.$, at $300 \mathrm{~K})$ and $E_{c}\left(E_{c \|} / E_{c \perp}=2.22\right.$, at $\left.300 \mathrm{~K}\right)$ values between the directions parallel and perpendicular to the cold-pressing axis.
\end{abstract}

\section{INTRODUCTION}

The formation of bismuth vanadate, $\mathrm{Bi}_{2} \mathrm{VO}_{5.5}(\mathrm{BiV})$, crystalline phase, which is a vanadium analog of an $n=1$ member of the Aurivillius family of oxides, has been reported in the $\mathrm{Bi}_{2} \mathrm{O}_{3}-\mathrm{V}_{2} \mathrm{O}_{5}$ binary system. ${ }^{1-4}$ It crystallizes in a non-centrosymmetric, polar orthorhombic class and is ferroelectric at room temperature. Systematic studies carried out, on both the single and polycrystalline samples, confirm that $\mathrm{BiV}$ exhibits two reversible phase transitions at 725 and $835 \mathrm{~K}^{5,6} \mathrm{BiV}$, by virtue of its high ionic conductivity in the high temperature tetragonal phase, has attracted the attention of many researchers for solid state fuel cell applications. ${ }^{7,8}$ However, the use of these ceramics for polar device applications is inhibited by its high dielectric loss ( 0.3 at $300 \mathrm{~K}$ and 3.5 at $725 \mathrm{~K}$, measured at $100 \mathrm{kHz}$ ), arising from its high ionic conductivity, as it hinders effective electrical poling. Our investigations into the effect of various dopants and additives on the physical properties of BiV ceramics have demonstrated that potassium chloride $(\mathrm{KCl})$ as an additive, is effective in modifying the microstructure as well as reducing the electrical conductivity. ${ }^{9}, 10$

Moreover, the electrical poling of the ceramic samples of the Aurivillius family of oxides is found to be ineffective in enhancing their piezoelectric response, since the rotation of the spontaneous polarization is confined to two dimensions, i.e., to the $a-b$ plane. In such ceramic samples, the piezoelectric properties can effectively be improved by the process of grain orientation, achieved

\footnotetext{
${ }^{a)}$ Address all correspondence to this author.
}

via techniques such as hot-pressing and hot-forging. The preferential grain orientation facilitates easier poling in the plane normal to the pressing direction. ${ }^{11-13}$ Our earlier investigations have revealed that the $\mathrm{KCl}$ addition, apart from considerably reducing the conductivity, leads to appreciable grain orientation $(75 \%)$ in $\mathrm{BiV}$ ceramics. ${ }^{10}$ Therefore, it is envisaged that these grainoriented ceramics with reduced electrical conductivity will exhibit better polar properties. In this paper, we report the results of our investigations pertaining to the structural and polar properties of $\mathrm{KCl}$ added, grainoriented, BiV ceramic samples.

\section{EXPERIMENTAL DETAILS}

\section{A. Sample preparation}

Bismuth vanadate polycrystalline powder was prepared by heating a stoichiometric mixture of bismuth oxide, $\mathrm{Bi}_{2} \mathrm{O}_{3}$ and vanadium pentoxide, $\mathrm{V}_{2} \mathrm{O}_{5}$ at $1070 \mathrm{~K}$ for $24 \mathrm{~h}$, with intermediate grinding and sintering steps. The formation of the desired phase was confirmed by x-ray powder diffraction studies (XRD). Prereacted BiV powders, along with known amounts of $\mathrm{KCl}$, were ballmilled for $2 \mathrm{~h}$ in an agate container. The oxide-salt mixture was cold-pressed and placed in a platinum crucible and heated to $1025 \mathrm{~K}$ for various durations. The ceramic samples thus obtained were studied for various physical properties, which are reported in this paper.

\section{B. Characterization}

The density of the sintered ceramic samples was determined by the liquid displacement method, using xylene as the liquid medium. The microstructure of the 
sintered ceramic samples and the average grain-size measurements were carried out using a Cambridge S360 scanning electron microscope (SEM). Quantitative elemental analysis was performed using LINK AN10000 energy dispersive x-ray analyzer (EDX), by employing ZAF-4 software. The formation of the desired phase, the lattice parameters, and the degree of grain orientation in the sintered ceramic samples were determined by XRD technique using $\mathrm{Cu} \mathrm{K}_{\alpha}$ radiation (Phillips PW 1050/70). The degree of $c$-axis orientation of the grains was estimated by employing the Lotgering's method, ${ }^{14}$ in which the orientation factor $(f)$ was calculated using the formula: $f=\left(p-p_{0}\right) /\left(1-p_{0}\right)$ where, $p=$ $\Sigma I_{00 l} / \Sigma I_{h k l}$ for the given oriented sample and $p_{0}=$ $\Sigma I_{00 l} / \Sigma I_{h k l}$ for the nonoriented sample. Differential thermal analyses (DTA) were performed on the powders, using a thermal analyzer STA 1500, Polymer Labs. The dielectric measurements (with an error not exceeding $\pm 5 \%$ ) were carried out on the gold sputtered and subsequently silver electroded samples. The anisotropy in the dielectric properties was studied by monitoring the dielectric constant along the directions parallel and perpendicular to the pressing axis. The capacitance, the dielectric loss, and the electrical conductivity were monitored, as a function of both frequency $(40 \mathrm{~Hz}$ to $100 \mathrm{kHz})$ and temperature (300 to $800 \mathrm{~K})$, using a Keithley 3330 multifrequency LCZ meter at a signal strength of $0.5 \mathrm{~V} \mathrm{rms.}$

The specimens for piezoelectric and pyroelectric measurements were poled at room temperature (RT) by applying a dc field of $15 \mathrm{kV} / \mathrm{cm}$ for $2 \mathrm{~h}$. The piezoelectric $d_{33}$ coefficient of the samples was measured using a Berlincourt $d_{33}$ meter, model CADT (range of measurement -20 to $1000 \mathrm{pC} / \mathrm{N}$ ) at $60 \mathrm{~Hz}$. Pyroelectric current was measured as a function of temperature (300 to $800 \mathrm{~K})$ by employing the direct method which was due to Byer-Roundy. ${ }^{15}$ A programmable furnace was used to heat the sample at a heating rate of $10{ }^{\circ} \mathrm{C} / \mathrm{min}$. A Keithley 485 autoranging picoammeter, with a storage facility, was used to record the pyroelectric current. The pyroelectric coefficient $p$ was evaluated from the relation $p=i /[A(d T / d t)]$ as a function of temperature, where $i$ is the pyroelectric current, $A$ is the electroded area, and $(d T / d t)$ is the heating rate. Ferroelectric hysteresis loop measurements were performed on electrically poled samples at a switching frequency of $50 \mathrm{~Hz}$, using a modified Sawyer-Tower circuit.

\section{RESULTS AND DISCUSSION}

\section{A. Microstructural analysis}

The density of the $10 \mathrm{~mol} \% \mathrm{KCl}$ added $\mathrm{BiV}$ ceramic sample is $\approx 84 \%$ of the theoretical value. It increases with decrease in the $\mathrm{KCl}$ content, but does not vary appreciably with increase in the sintering time.
Figures 1(a) and 1(b) show the scanning electron micrographs obtained for the pure and $10 \mathrm{~mol} \% \mathrm{KCl}$ added $\mathrm{BiV}$ ceramics. It is evident from the microstructure that the $\mathrm{KCl}$ addition enhances the grain growth. Indeed, the average $\mathrm{BiV}$ grain size increases steadily with increase in the amount of $\mathrm{KCl}$ and the grain-morphology changes from symmetrical to lenticular [Fig. 1(b)]. The presence of interpenetrating grains is a clear indication of two-dimensional grain growth, which progresses by partial absorption between grains during sintering. The occurrence of abnormal grain growth, as a consequence of secondary recrystallization, could be due to the presence of a small amount of liquid phase (flux) formed by the additive at the grain boundaries. ${ }^{16,17}$ Systematic EDX analyses carried out on these samples indicate that the $\mathrm{KCl}$ distribution is nonuniform, residing primarily at the grain boundaries [Fig. 2(a)]. Prismaticshaped crystallites of $\mathrm{KCl}$ are also present at the grain boundaries, as shown in Fig. 2(b). The possibility of $\mathrm{K}^{+}$
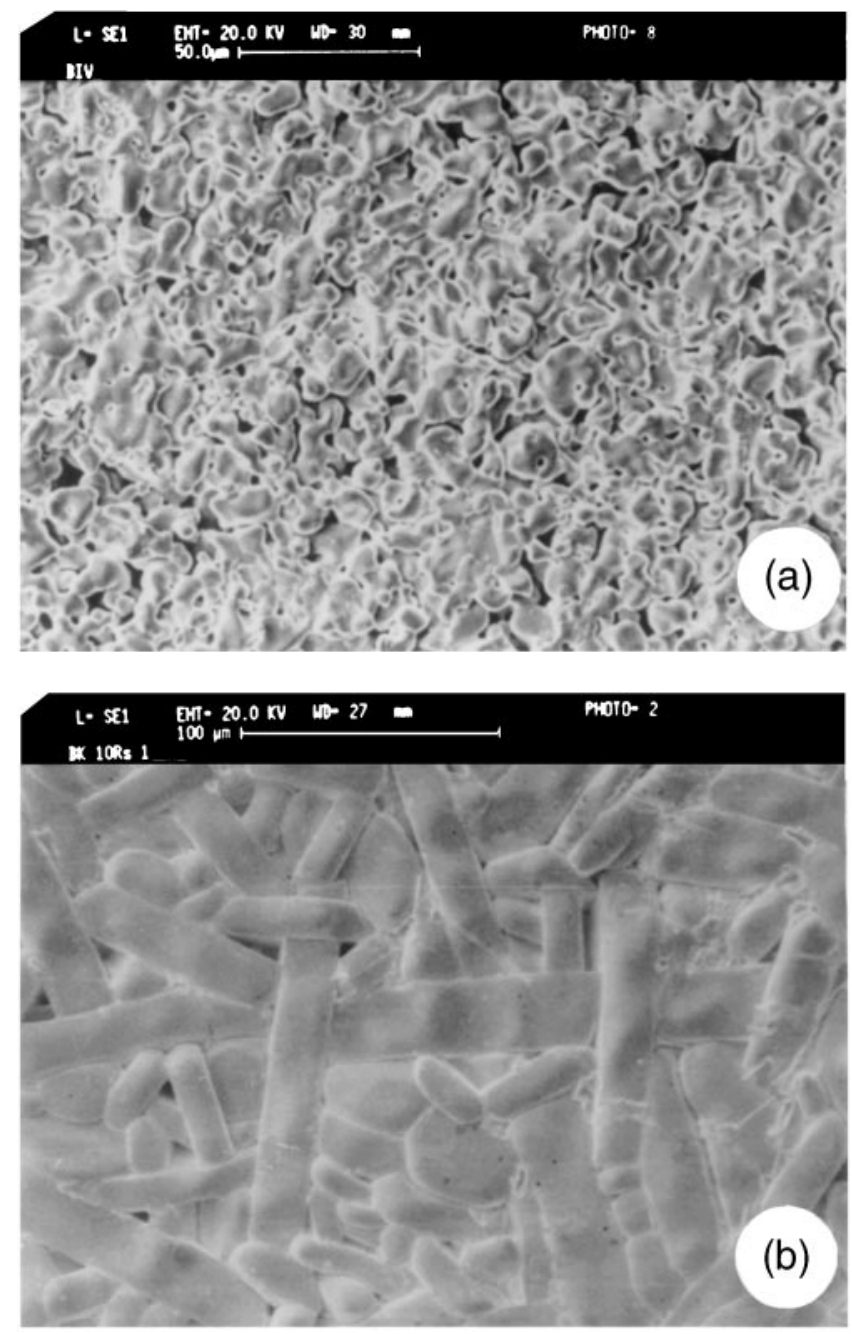

FIG. 1. Scanning electron micrographs obtained for (a) additive-free and (b) $10 \mathrm{~mol} \% \mathrm{KCl}$ added $\mathrm{BiV}$ ceramics. 

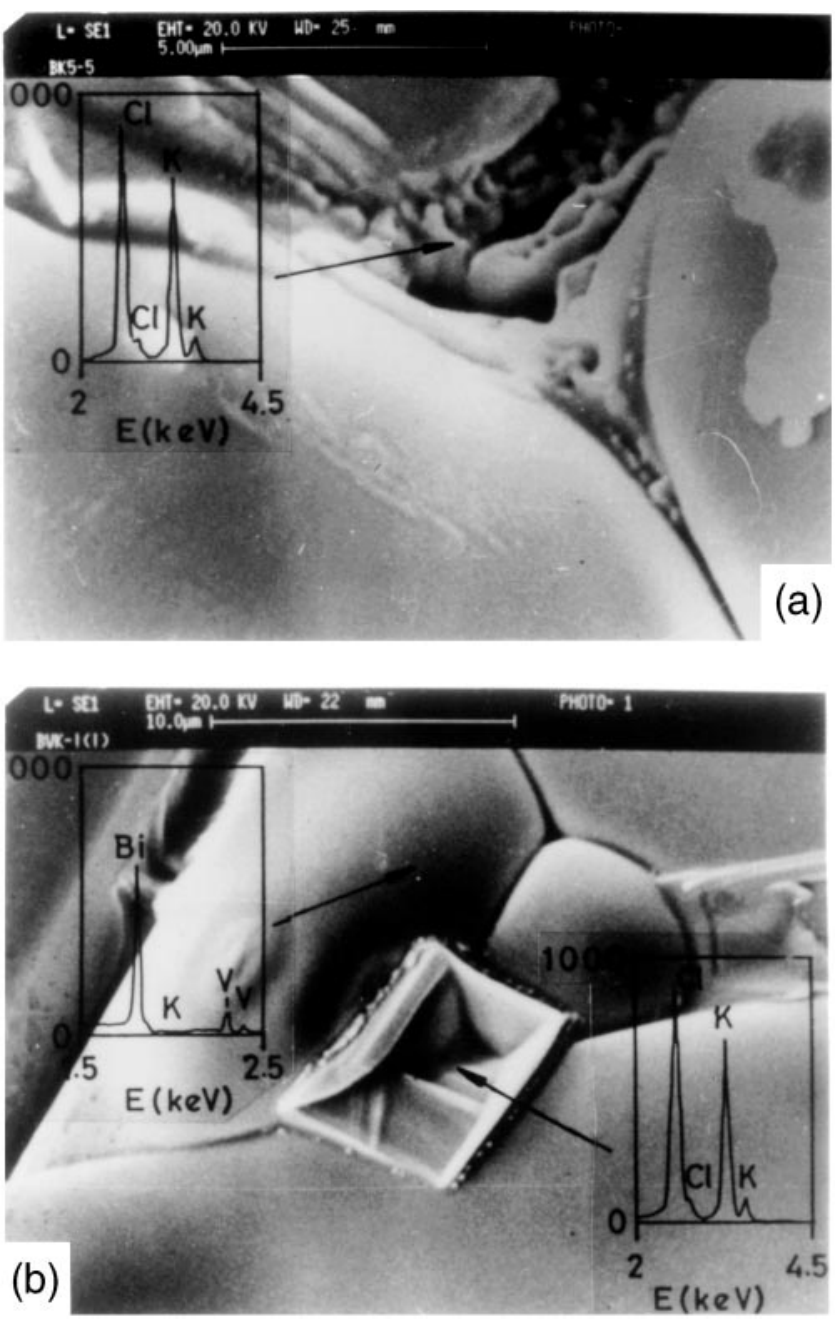

FIG. 2. Scanning electron micrographs obtained for the KCl-modified BiV ceramics: (a) shows the grain-boundary region, with the EDX pattern as the inset, and (b) shows the $\mathrm{KCl}$ crystallite at the grain boundary along with the EDX pattern.

ions entering $\mathrm{Bi}^{3+}$ sites with the formation of oxygen vacancies is not completely ruled out, since their ionic radii are comparable. However, the lattice parameters of $\mathrm{KCl}$ added ceramics [i.e., $a=16.596(3), b=5.595(1)$, and $c=15.299(3) \AA$ for the $10 \mathrm{~mol} \% \mathrm{KCl}$ containing sample] are not significantly different from those of additive-free $\operatorname{BiV}[a=16.594(3), b=5.602(1)$, and $c=15.297(3) \AA]$. Thus, it is clear that the relatively pure $\mathrm{BiV}$ regions form the core of the grains, and the grain-boundary regions rich in $\mathrm{KCl}$ form the grainshell regions. Transmission electron microscopic (TEM) studies are in progress to substantiate the existence of core-shell grain structure. The presence of elongated grains with large aspect ratio suggests the possible occurrence of grain orientation in these ceramics. Indeed, the XRD patterns recorded for the ceramic disks suggest the existence of grain orientation, the details of which are elucidated in the subsequent section.
Grain growth in ceramics is known to be strongly dependent on the sintering temperature and the soaking duration. ${ }^{16}$ In the present case, since sintering at temperatures above $1025 \mathrm{~K}$ yielded low density, porous samples, the sintering temperature was fixed at $1025 \mathrm{~K}$ and the soaking time was varied in order to visualize its influence on the grain morphology, grain orientation, and the resultant change in the physical properties. There is a large increase in the grain size with increase in the sintering time. The average grain size is not computed for these samples, since there is a large distribution of grain sizes in these samples, due to secondary recrystallization. The corresponding increase in the grain orientation was monitored via XRD studies. Figures 3(a) and 3(b) show the scanning electron micrographs obtained for the perpendicular and parallel faces of the $10 \mathrm{~mol} \% \mathrm{KCl}$ added sample with $f=0.75$
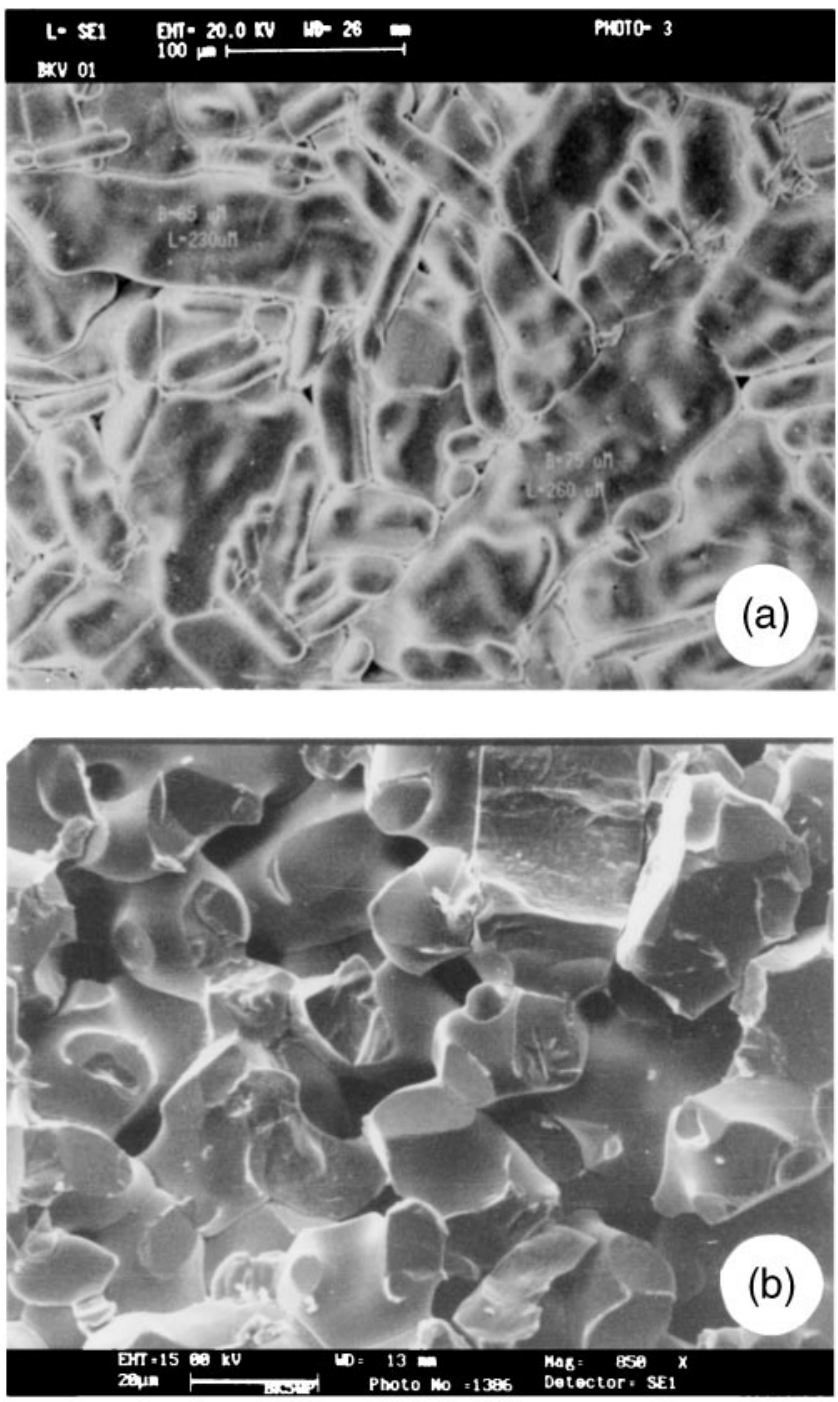

FIG. 3. SEM micrographs recorded on the (a) perpendicular and (b) parallel surfaces, of the pressing axis, for the KCl-modified grain-oriented ceramic $(f=0.75)$. 
(sintered at $1025 \mathrm{~K}$ for $60 \mathrm{~h}$ ). Large elongated grains, with plate-like morphology, which are piled up along the pressing direction, are seen on the perpendicular surface, whereas more symmetrical grains are found on the parallel surface.

The SEM studies carried out on the chemically etched $\left[\mathrm{HNO}_{3}: \mathrm{H}_{2} \mathrm{O}(1: 4)\right] \mathrm{KCl}$-modified $\mathrm{BiV}$ ceramics, revealed the presence of ferroelectric domains. These domains are confined mainly to the central regions of the grains, implying the ferroelectric nature of the core and the paraelectric nature of the shell of the grains [Fig. 4(b)]. The domain pattern, obtained for the additivefree BiV ceramic sample, is also shown in Fig. 4(a) for comparison. It is clear from the micrographs that the domain pattern of the $\mathrm{KCl}$-modified $\mathrm{BiV}$ sample is strikingly different from that of the additive-free BiV sample. The domain structure of the additive-free BiV ceramic has normal stripe-like morphology, stripes extending from grain to grain, which is typical of a long range ordered ferroelectric state. Whereas, in the $\mathrm{KCl}$-modified samples, the domain density increases, the domain size is finer and the domain boundaries are irregular and "wavy" [Fig. 4(b)]. The occurrence of a similar "wavy" domain structure has been reported ${ }^{18,19}$ in lead potassium zirconate (PKZT) ceramics and is attributed to the domain-wall pinning due to mobile defects. A small amount of the additive is found to have a remarkable influence on the domain structure of BiV. The density and the "waviness" of the domains increase significantly, which is indicative of domain-wall pinning. The evolution of such a domain structure can be explained as follows. As the sample is cooled from its high temperature paraelectric state to the ferroelectric state, the polarized defect complexes $\left(\mathrm{K}^{+}\right.$-oxygen vacancy dipoles) can remain mobile till significantly low temperatures. Consequently, during the nucleation and growth of domains, these defect complexes can preferentially segregate at the domain boundaries, resulting in polarization-pinning. Domain-pinning can lead to a continuous bending in the domain orientation between various equivalent polar directions, giving rise to a "wavy" domain pattern. The influence of such a domain morphology on the polar properties of these samples is discussed in the subsequent sections.

\section{B. XRD analysis}

The XRD pattern recorded for the crushed powders of the KCl-modified BiV sample clearly shows the Bragg peaks corresponding to the $\mathrm{BiV}$ and the $\mathrm{KCl}$ phases, indicating that $\mathrm{KCl}$ crystallites are dispersed in the BiV matrix. ${ }^{10}$ The XRD patterns recorded for the face perpendicular to the pressing axis of the ceramic disk exhibit intense $(00 l)$ and weak $(h k l)$ lines. The intensity of the $(00 l)$ peaks increases with increase in the amount of $\mathrm{KCl}$ used during sintering [Figs. 5(b) and 5(c)]. For comparison, the XRD pattern recorded for the randomly oriented additive-free $\mathrm{BiV}$ ceramic is shown in Fig. 5(a). These results indicate that the BiV grains are oriented with their $c$-axes along the pressing direction. The degree of grain orientation in these samples was determined, using the Lotgering method, based on the data recorded over a $2 \theta$ range of 10 to $60^{\circ}$. The degree of grain orientation $(f)$ increases with increase in the $\mathrm{KCl}$ amount (Fig. 6). The value of $f$ could also be effectively enhanced by increasing the sintering duration (with intermediate grinding steps). Fig. 7 shows the increase in $f$ with increase in the sintering duration for $10 \mathrm{~mol} \% \mathrm{KCl}$ added sample. Thus, a degree of grain orientation as high as $75 \%$ could be achieved by sintering the $10 \mathrm{~mol} \% \mathrm{KCl}$ added sample at $1025 \mathrm{~K}$ for $60 \mathrm{~h}$.

The lattice strain was computed by monitoring the line broadening and line shift of a few selected Bragg peaks, according to the procedure outlined in our earlier paper. ${ }^{10}$ When $\mathrm{BiV}$ is cooled below the transition temperature (tetragonal $\rightarrow$ orthorhombic), the volume change associated with the phase transformation will
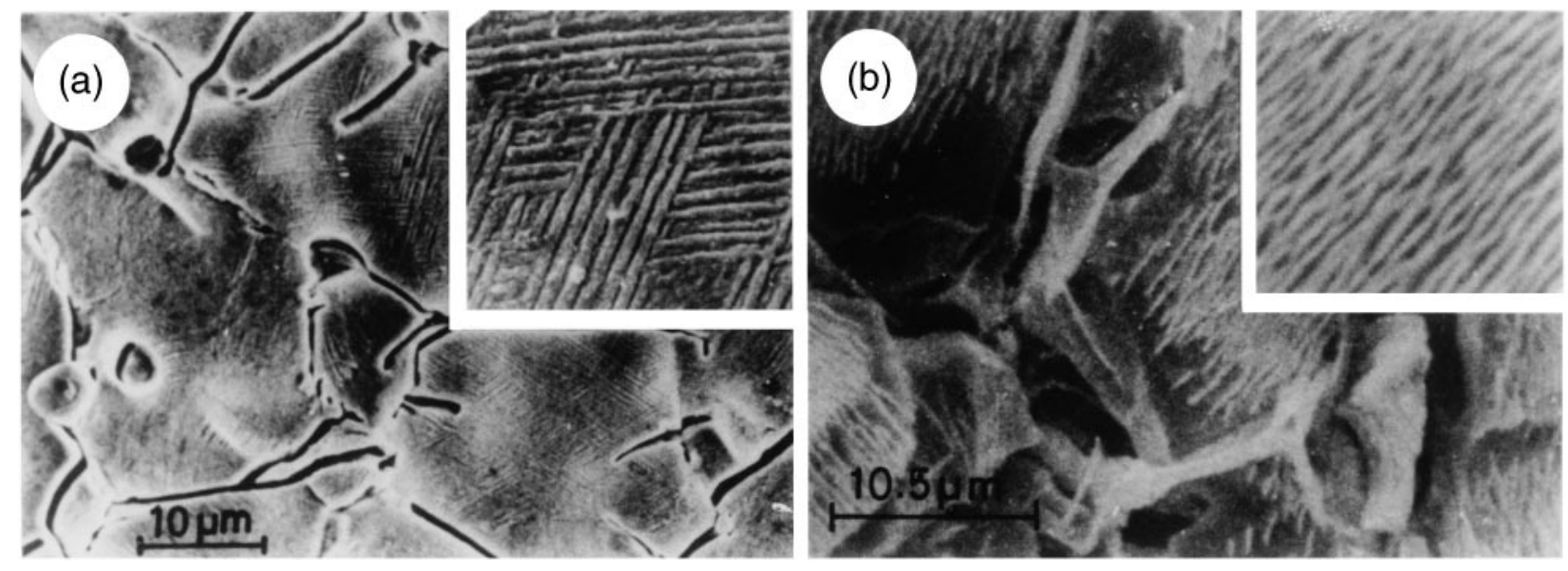

FIG. 4. Scanning electron micrographs of the chemically etched samples of (a) additive-free and (b) KCl-modified BiV ceramics. 


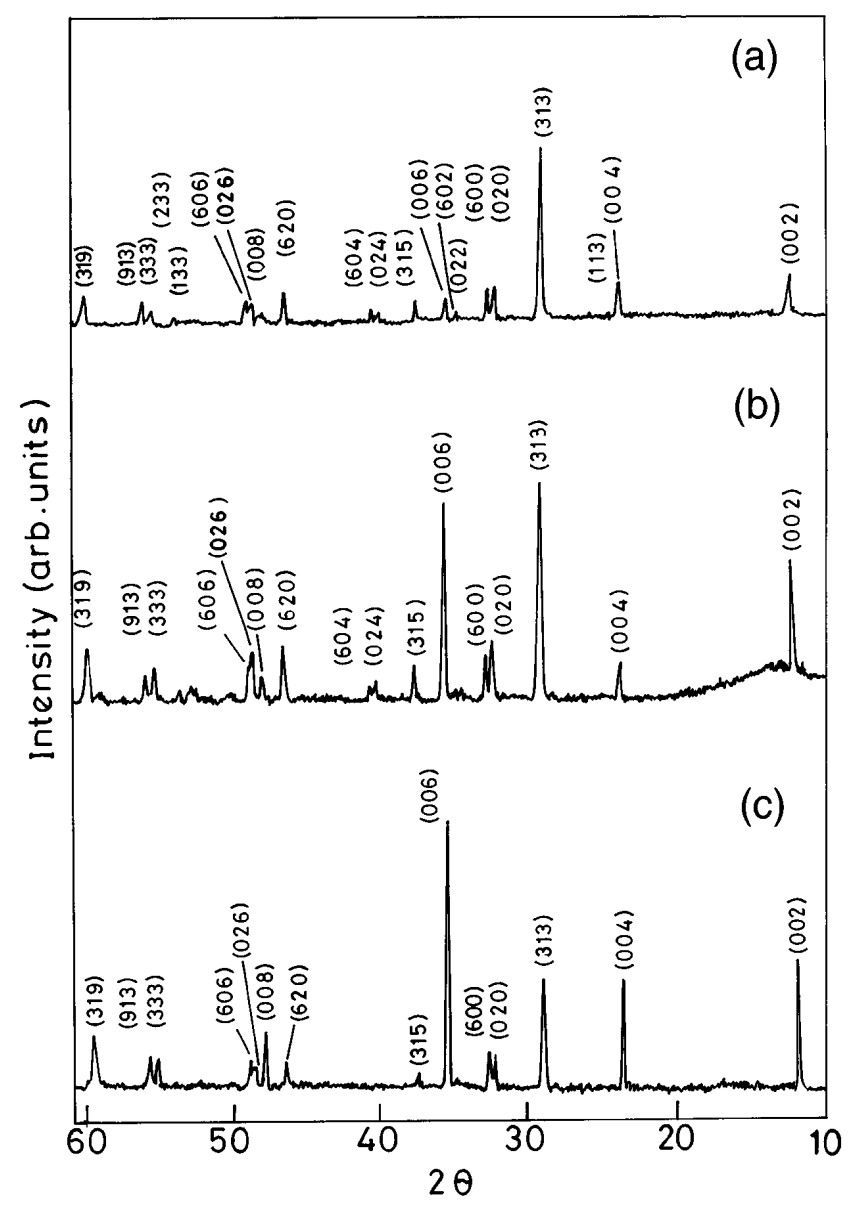

FIG. 5. X-ray powder diffraction patterns recorded for the perpendicular face of the (a) additive-free, (b) $2 \mathrm{~mol} \%$, and (c) $10 \mathrm{~mol} \% \mathrm{KCl}$ added BiV ceramic disks.

eventually generate a slight lattice deformation of its constituent orthorhombic $\mathrm{BiV}$ crystallites. It is because of the fact that the volume change for one crystallite can be inhibited by the surrounding crystallites. In the present case, since almost all the grain boundaries consist of $\mathrm{KCl}$ crystallites of varied sizes, it is more likely that it can create an additional lattice strain. The lattice strain could also be induced as a result of the lattice mismatch between the core and the shell of the grains, which is likely to arise as a consequence of the limited solubility of $\mathrm{KCl}$ in the core and its preferential segregation at the shell of the grains. The lattice strain computed for the $10 \mathrm{~mol} \% \mathrm{KCl}$ added sample is found to be higher than that for the additive-free sample. ${ }^{10}$

\section{Dielectric properties}

The dielectric constant $\left(\epsilon_{r}\right)$ and the dielectric loss $(\tan \delta)$ at room temperature, measured along both the parallel $\left(\epsilon_{r \|}\right.$ and $\left.\tan \delta_{\|}\right)$and the $\left(\epsilon_{r \perp}\right.$ and $\left.\tan \delta_{\perp}\right)$ directions to the pressing axis, for the ceramic sample with $f=0.75$, decrease with increase in frequency [Figs. 8(a) and 8(b)].

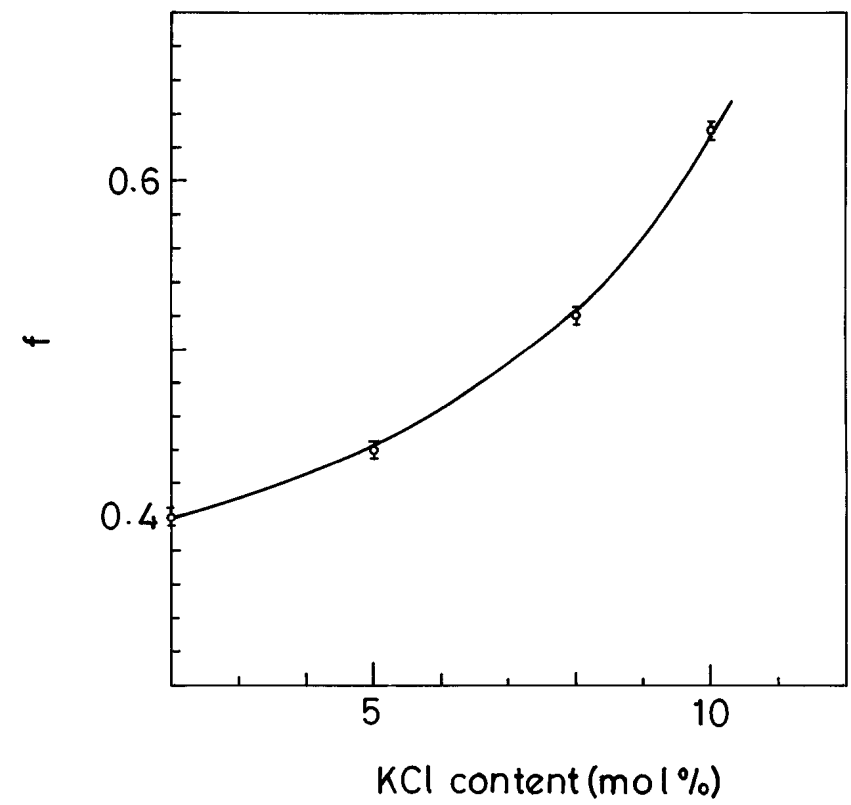

FIG. 6. Variation of $f$ with increase in the $\mathrm{KCl}$ content.

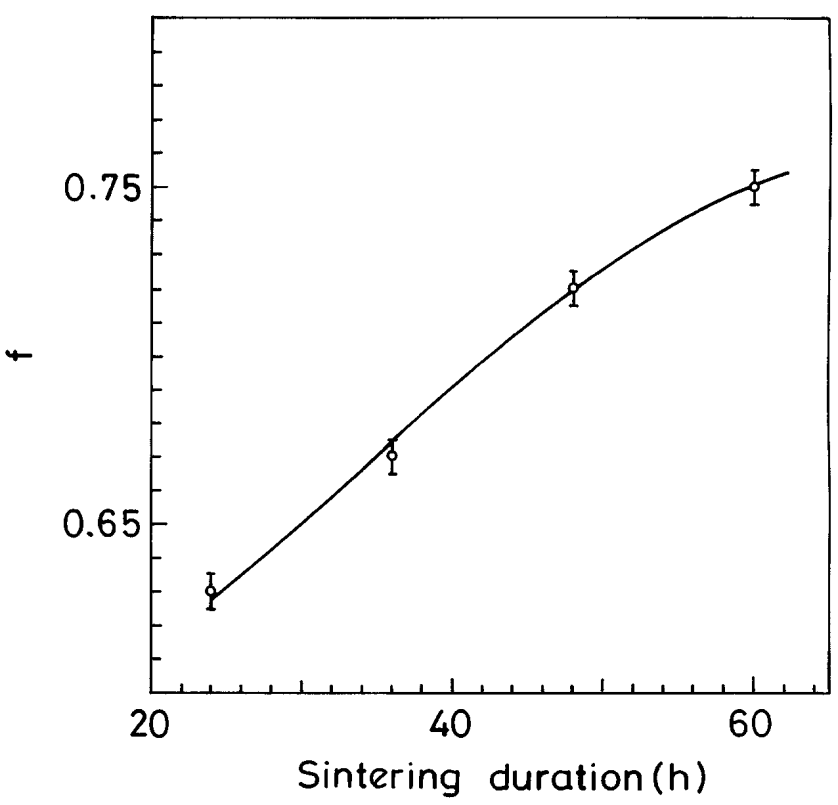

FIG. 7. Variation of $f$ as a function of the sintering time for the $10 \mathrm{~mol} \% \mathrm{KCl}$ added $\mathrm{BiV}$ ceramic.

Both $\epsilon_{r \perp}$ and $\tan \delta_{\perp}$ are higher than $\epsilon_{r \|}$ and $\tan \delta_{\|}$over the whole frequency range covered, suggesting that the polar axis lies in the $a-b$ plane. In fact, the polar axis of BiV single crystals has been confirmed to be the crystallographic $a$-axis, by dielectric and ferroelectric studies. ${ }^{20}$ Therefore, the dielectric constant along any axis in the a-b plane is expected to be higher than that measured normal to the $a-b$ plane. In the grainoriented ceramics, since most grains are aligned with their $c$-axes along the pressing direction, the dielectric 

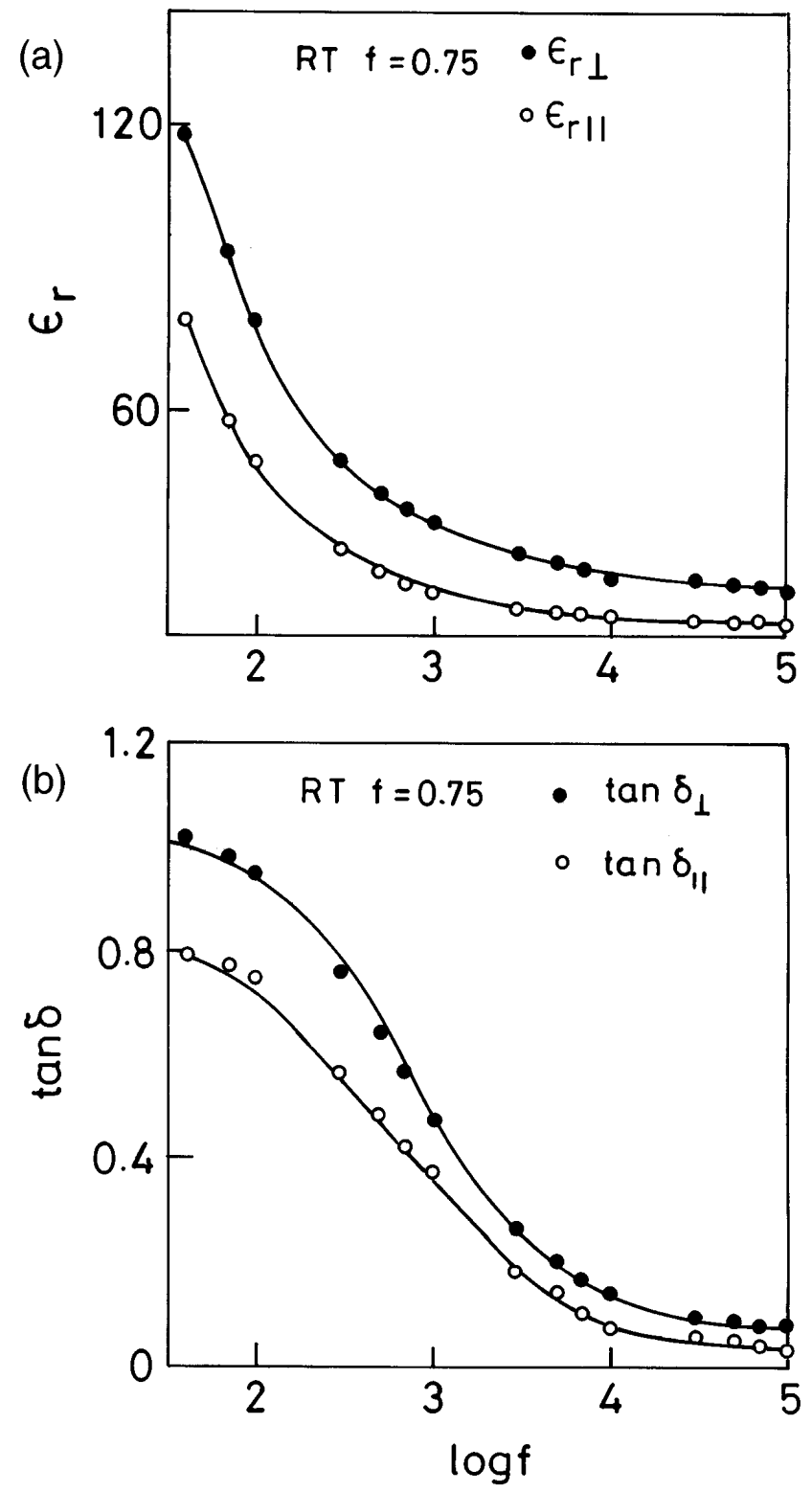

FIG. 8. Variation of (a) $\epsilon_{r \|}$ and $\epsilon_{r \perp}$ and (b) $\tan \delta_{\|}$and $\tan \delta_{\perp}$, with frequency, at $300 \mathrm{~K}$ for the $\mathrm{KCl}$-modified grain-oriented ceramic $(f=0.75)$.

constant measured along the direction perpendicular to the pressing axis is higher. Figures 9(a) and 9(b) show the temperature dependence of $\epsilon_{r}$ of the KCl-modified grain-oriented sample $(f=0.75)$ monitored at various frequencies. It is evident from this figure that there is no measurable shift in the temperature of dielectric maximum $\left(T_{c}\right)$ with the frequency. The variation of $\epsilon_{r \perp}$ and $\epsilon_{r \|}$ along with the $\epsilon_{r}$ of the randomly oriented additive-free $\mathrm{BiV}$ sample, as a function of temperature, is shown in Fig. 10. A significant anisotropy in the dielectric constant and the dielectric loss, between the parallel and the normal directions of the pressing axis, is observed. The ratio of $\epsilon_{r \perp}$ to $\epsilon_{r \|}$ increases with
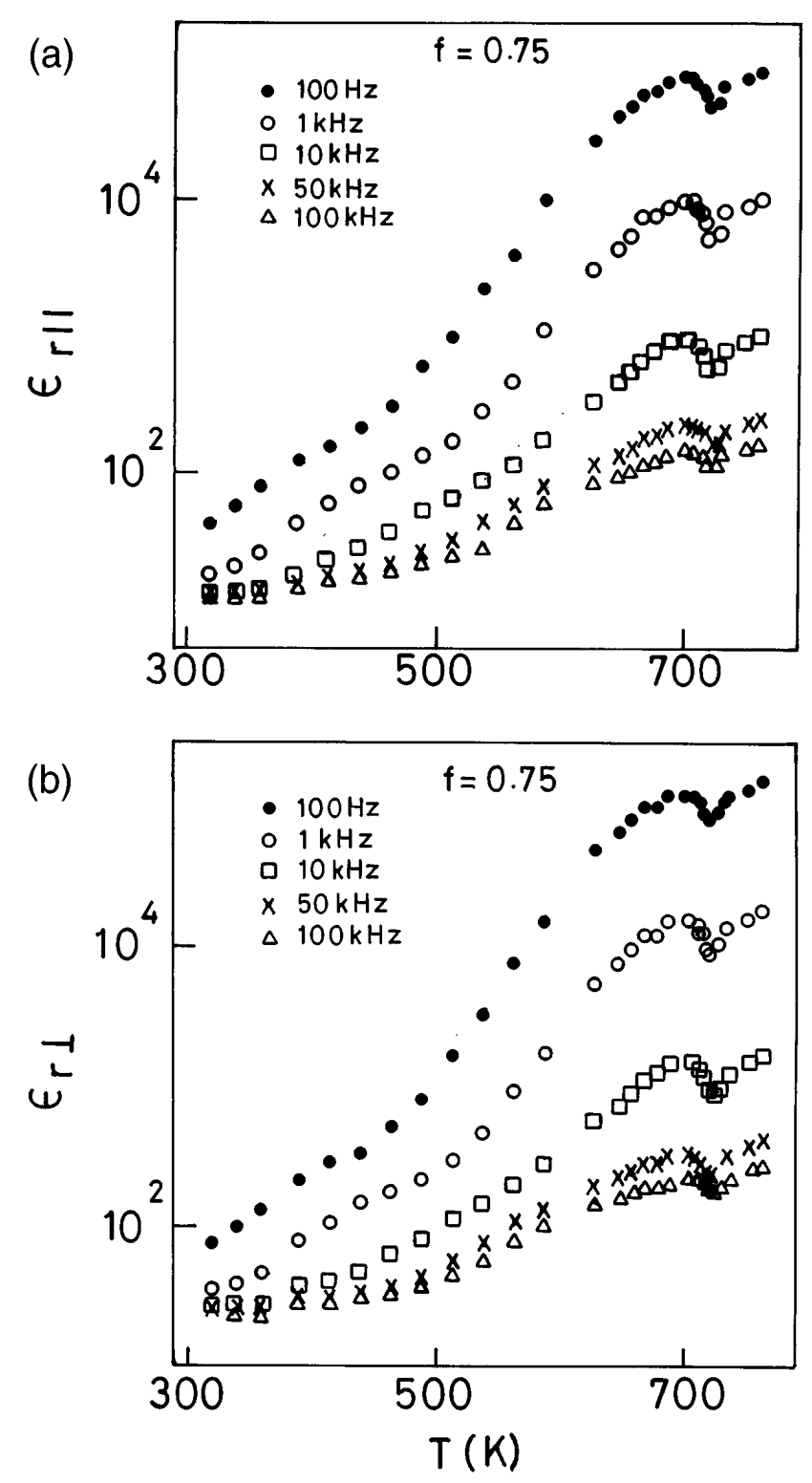

FIG. 9. Temperature dependence of (a) $\epsilon_{r \|}$ and (b) $\epsilon_{r \perp}$ for the $\mathrm{KCl}$-modified grain-oriented ceramic at various frequencies.

increase in temperature and reaches a maximum at the Curie temperature $T_{c}$ (Table I). Both $\epsilon_{r \perp}$ and $\epsilon_{r \|}$ show a maximum at the same temperature, which is close to the $T_{c}$ estimated based on DTA. The $T_{c}$ obtained for the $\mathrm{KCl}$-modified sample is lower than that of the additive-free sample. The decrease in $T_{c}$ is attributed to the decrease in the orthorhombic distortion (the b/a ratio) associated with the sample. The $\epsilon_{r}$ and $\tan \delta$ are much lower for the $\mathrm{KCl}$-modified $\mathrm{BiV}$ ceramics than those for the additive-free $\mathrm{BiV}$, both at $\mathrm{RT}$ and at $T_{c}$. This is attributed to the presence of $\mathrm{KCl}$ crystallites of lower dielectric constant values dispersed in the $\mathrm{BiV}$ matrix. Also, the higher porosity and the increased resistivity (as a result of reduced grain-grain contact) would certainly 


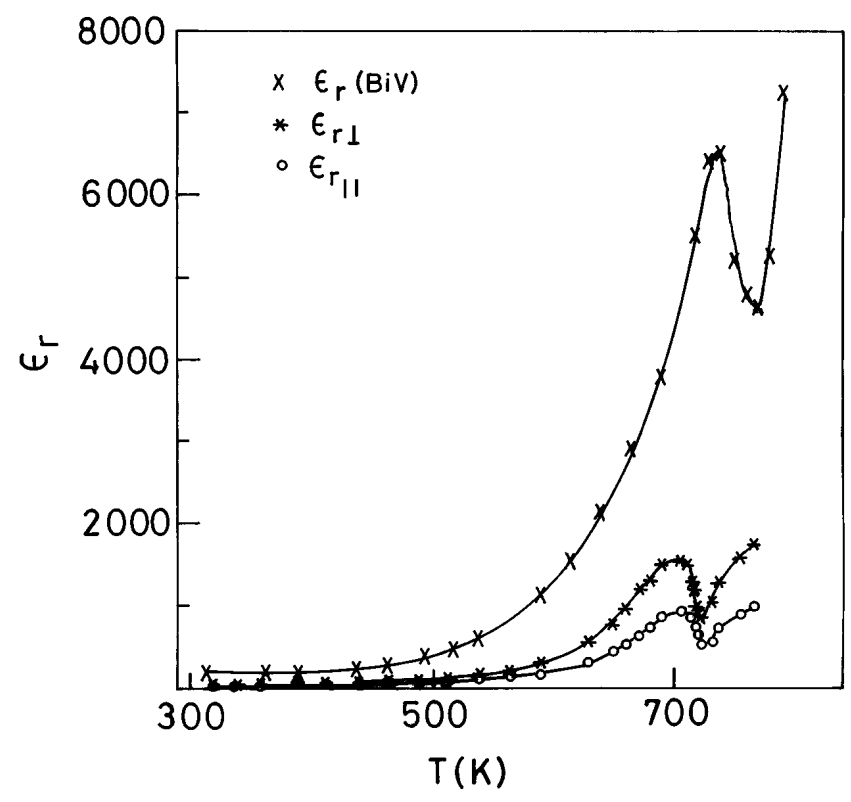

FIG. 10. Temperature dependence of $\epsilon_{r \|}$ and $\epsilon_{r \perp}$ for KCl-modified and $\epsilon_{r}$ of the additive-free $\mathrm{BiV}$ sample, measured at $10 \mathrm{kHz}$.

TABLE I. Dielectric and conductivity anisotropies of BiV single crystal and KCl-modified grain-oriented ceramic.

\begin{tabular}{lccl}
\hline \hline \multicolumn{1}{c}{ Sample } & $\frac{\sigma_{\perp}}{\sigma_{\|}}(300 \mathrm{~K})$ & $\frac{\epsilon_{r \perp}}{\epsilon_{r \|}}(300 \mathrm{~K})$ & $\frac{\epsilon_{r \perp}}{\epsilon_{r \|}}\left(T_{c}\right)$ \\
\hline BiV single crystals & 10 & 2.2 & 3.5 \\
KCl-modified BiV ceramic & 3.51 & 1.5 & 2.76 \\
\hline \hline
\end{tabular}

play a crucial role in reducing the dielectric constant. Another possible mechanism could be the restriction imposed on the domain-wall movement, as a consequence of the domain wall-pinning, confinement of the domains to the core region of the grains, and the presence of the secondary phase at the grain boundaries.

The KCl-modified BiV sample exhibits a broad and depressed phase transition, with no frequency dispersion. In order to verify whether the observed phenomenon is a diffused phase transition (DPT), ${ }^{21}$ the following analysis was adopted: The temperature dependence of the dielectric constant at any temperature $T>T_{c}$ is governed by the equation, $\left[1 / \epsilon_{r}-1 / \epsilon_{r(\max )}\right]=A\left(T-T_{c}\right)^{\tau}$, where the exponent $\tau$ indicates the amount of diffuseness. The value of $\tau$ is equal to 1 for a typical sharp transition (Curie-Weiss type of transition), it has a value between 1 and 2 for a DPT, and is equal to 2 for a complete diffused phase transition. ${ }^{22,23}$ To estimate the value of $\tau$, a plot of $\log \left[1 / \epsilon_{r}-1 / \epsilon_{r(\max )}\right]$ vs $\log \left[T-T_{c}\right]$ is generated [Figs. 11(a) and 11(b)] for the $10 \mathrm{~mol} \% \mathrm{KCl}$ added ceramic. The curve does not show a linear behavior and the slope changes continuously, indicating that $\tau$ decreases with increase in temperature. Thus, it becomes difficult to estimate the value of the exponent.
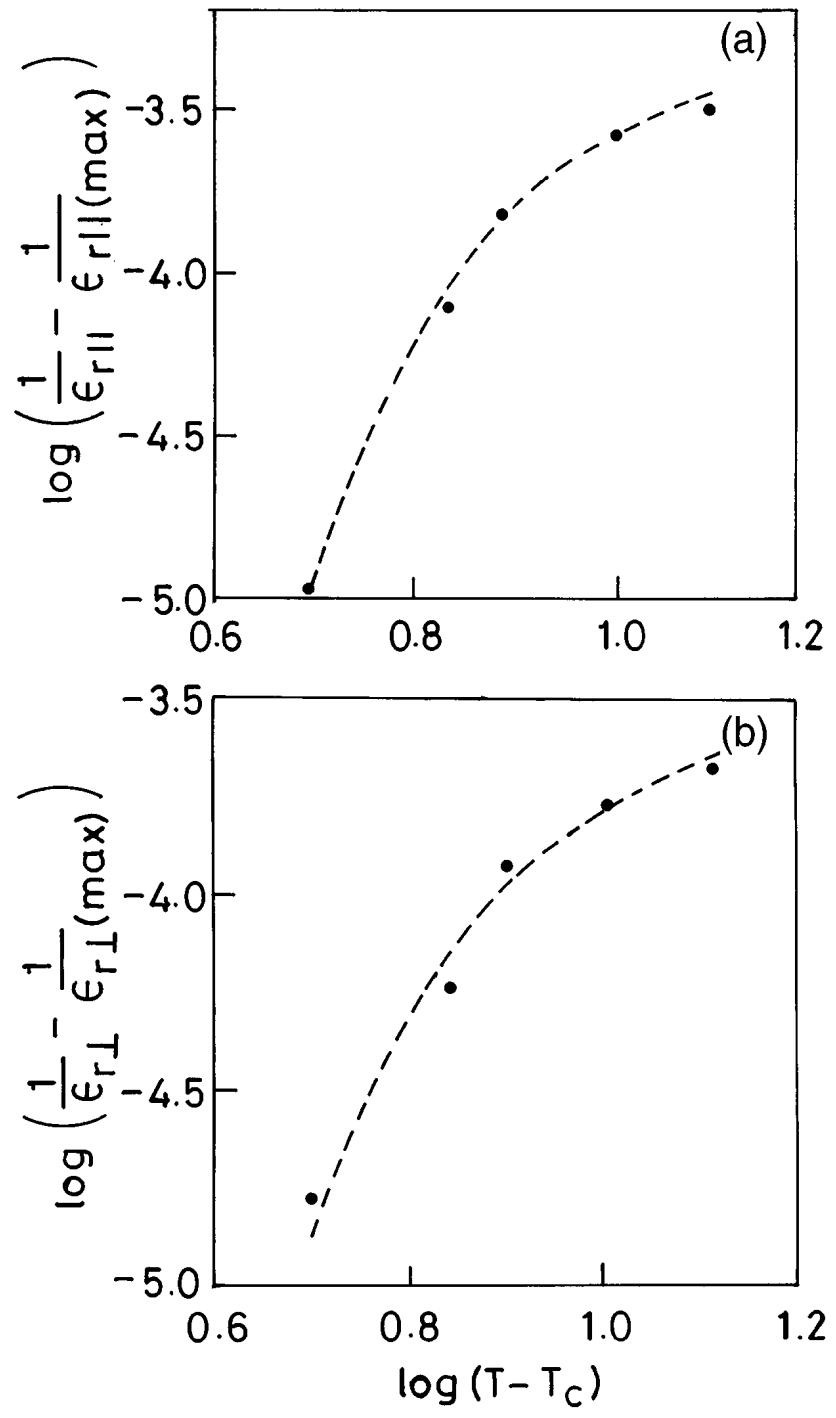

FIG. 11. $\log \left[1 / \epsilon_{r}-1 / \epsilon_{r(\max )}\right]$ vs $\log \left[T-T_{c}\right]$ at $10 \mathrm{kHz}$ for (a) $\epsilon_{r \|}$ and (b) $\epsilon_{r \perp}$.

Alternatively, in order to examine the validity of the Curie-Weiss law in the vicinity of the $T_{c}$, the inverse of dielectric constant (both $\epsilon_{r \|}$ and $\epsilon_{r \perp}$ ) is plotted as a function of temperature [Figs. 12(a) and 12(b)]. It is evident from the plot that the Curie-Weiss law is obeyed only above a certain temperature, $T_{c 1}$ and a significant deviation from the linear behavior is observed in the vicinity of $T_{c}$. The parameter $\Delta_{T}=T_{c 1}-T_{c}$ is known to be a measure of the thermal diffuseness. ${ }^{24}$ The finite value of $\Delta_{T}$, associated with the transition for the $\mathrm{KCl}$ modified BiV samples, indicates the deviation from the Curie-Weiss behavior. Therefore, the ferro-paraelectric transition, exhibited by the $\mathrm{KCl}$-modifed $\mathrm{BiV}$ samples, can be classified as DPT, in contrast with the normal Curie-Weiss type of transition exhibited by the additivefree BiV sample. ${ }^{20}$ Since it is known that the formation of core-shell grain structure influences the nature of 

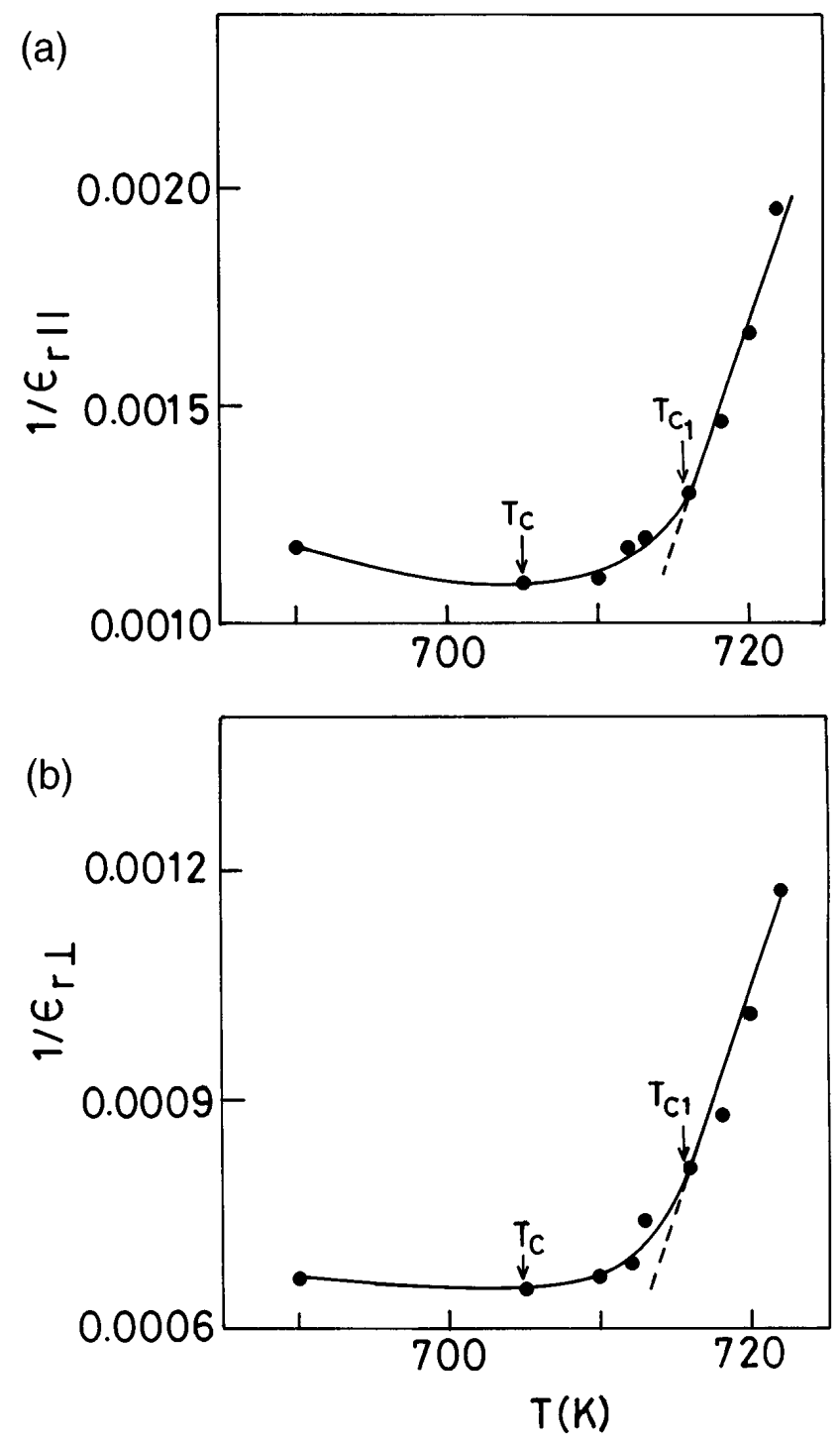

FIG. 12. Reciprocal of dielectric constant (a) $\epsilon_{r \|}$ and (b) $\epsilon_{r \perp}$ plotted as a function of temperature, for the $\mathrm{KCl}$-modified $\mathrm{BiV}$ sample, at $10 \mathrm{kHz}$.

the phase transition, ${ }^{25}$ the DPT exhibited by the $\mathrm{KCl}$ modified samples may be attributed to the presence of core-shell-like grain structure formed by the grainboundary segregated $\mathrm{KCl}$. It is understood that the internal stress present in the ceramic samples leads to diffused phase transitions. ${ }^{26}$ It is clear from the earlier discussion that the lattice strain estimated for the $10 \mathrm{~mol} \% \mathrm{KCl}$ added sample is higher than that for the additive-free sample, which could also give rise to the observed change in the nature of the phase transition.

\section{Resistivity measurements}

The resistivity of the $\mathrm{KCl}$-modified $\mathrm{BiV}$ ceramic samples is found to be higher than that of the additivefree BiV sample. The grain and grain-boundary contri- butions to the resistance of the sample could be resolved by analyzing the impedance spectra. The grain-boundary resistance of the $\mathrm{KCl}$-modified sample is more than two orders of magnitude higher than that of the additive-free BiV sample. ${ }^{10}$ For the grain-oriented ceramic samples, the conductivity data were measured in the two perpendicular directions and found that $\sigma_{\perp}$ is higher than $\sigma_{\|}$. The anisotropy in conductivity $\left(\sigma_{\perp} / \sigma_{\|}\right)$is $=3.51$ at $300 \mathrm{~K}$, for the ceramic with $f=0.75$ (Table I). Similar results have been reported in other bismuth layer compounds such as $\mathrm{PbBi}_{2} \mathrm{Nb}_{2} \mathrm{O}_{9}, \mathrm{SrBi}_{4} \mathrm{Ti}_{4} \mathrm{O}_{15}$, etc. ${ }^{11}$ The $c$-axis oriented single crystalline plate of $\mathrm{BiV}$ shows a large anisotropy $(10: 1$, at $300 \mathrm{~K})$ in the electrical conductivity, between the directions normal and parallel to the $c$-axis, due to its layered nature. ${ }^{27}$ Since most of the $\mathrm{BiV}$ grains are oriented with their $c$-axes along the pressing direction, in the $\mathrm{KCl}$-modified $\mathrm{BiV}$ ceramic, the lower conductivity along the pressing direction is justifiable.

\section{E. Piezoelectric properties}

The KCl-modified samples poled at $15 \mathrm{kV} / \mathrm{cm}$ for $4 \mathrm{~h}$ at RT are found to be piezoactive in contrast with the additive-free BiV ceramics. The piezoelectric $d_{33}$ coefficients are measured in the directions both parallel and perpendicular to the pressing axis for the samples poled under identical conditions, in the direction of the measurement. The $\mathrm{KCl}$-modified grain-oriented $\mathrm{BiV}$ ceramic with $f=0.75$, exhibits significant $d_{33}$ values of $53 \mathrm{pC} / \mathrm{N}$ and $38 \mathrm{pC} / \mathrm{N}$ in the directions perpendicular and parallel to the pressing directions, respectively. The piezoelectric coefficients of the samples with lower degree of grain orientation are small (smaller than the measurement range of the $d_{33}$ meter used) and hence could not be quantified.

\section{F. Pyroelectric properties}

The pyroelectric measurements are carried out on the grain-oriented samples in the two perpendicular directions, maintaining identical poling conditions prior to the two measurements. Figure 13 shows the temperature variation of the pyroelectric coefficient $(p)$ measured in the two directions, for the sample with $f=0.75$. A significant anisotropy is found in the pyroelectric response in the two directions, $p_{\perp}$ being higher than $p_{\|}$. In both directions, $p$ is positive and shows a broad peak near the transition temperature, then decreases and subsequently becomes negative. It is clear that the anisotropy increases with increase in temperature and undergoes a maximum at the transition temperature (the ratio $p_{\perp} / p_{\|}$is 3.33 at $\mathrm{RT}$ and 25 at $T_{c}$ ). These data, along with the pyroelectric figure of merit for the quick evaluation of the material, given by $f_{Q}=p / \epsilon_{0} \times$ $\epsilon_{r}$, calculated based on the dielectric constant and the 


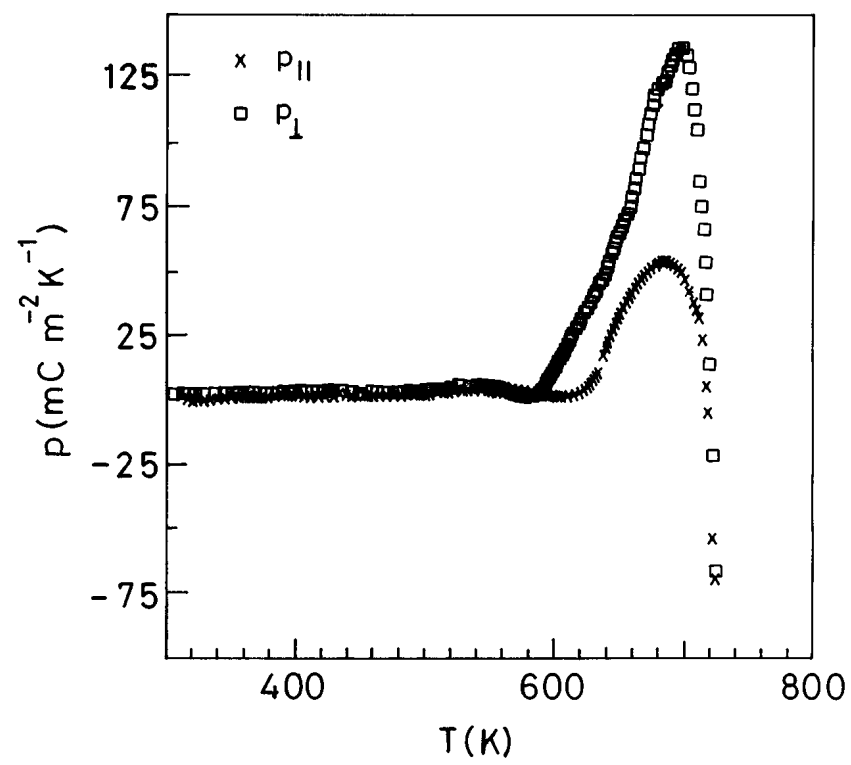

FIG. 13. Temperature dependence of $p_{\|}$and $p_{\perp}$ for the KCl-modified grain-oriented ceramic.

pyroelectric coefficient, are listed in Table II. Although, the $p$ values for the $\mathrm{KCl}$-modified $\mathrm{BiV}$ samples are lower than that reported for the additive-free $\mathrm{BiV}$ ceramic samples, ${ }^{20}$ they have better $f_{Q}$ values because of their lower dielectric constant values.

The positive sign of the pyroelectric coefficient is unexpected for a ferroelectric material. In ferroelectric materials, the primary coefficient is large and negative and the secondary coefficient is small and generally has positive sign, but may take either sign depending on the values of the coefficients. The measured pyroelectric coefficient (at constant stress) is the sum of the two terms and is given by $p_{i}^{\sigma}=p_{i}^{\epsilon}+\left(d_{i j k}\right)^{T}\left(C_{j k l m}\right)^{T, E}\left(\alpha_{l m}\right)^{\sigma}$ where $p_{i}^{\sigma}$ is the total pyroelectric coefficient measured at constant stress, and $p_{i}^{\epsilon}$ is the pyroelectric effect at constant strain and represents the primary effect $\left(p_{p r i}\right)$. The second term, which involves the piezoelectric tensor $\left(d_{i j k}\right)^{T}$ at constant temperature, the elastic stiffness coefficient, $\left(C_{j k l m}\right)^{T, E}$, at constant temperature and electric field and the thermal expansion coefficient $\left(\alpha_{l m}\right)^{\sigma}$ at constant stress represents the secondary effect $\left(p_{\text {sec }}\right)$.
Thus, the measured pyroelectric coefficient, which involves both the contributions, is generally negative for ferroelectric materials. The positive sign of $p$, in the present case, indicates that the $p_{s e c}$, which is piezoelectric in origin, is larger than $p_{p r i}$ in this temperature range $(300-725 \mathrm{~K})$. In the additive-free $\mathrm{BiV}$ ceramic sample, it is noticed that $p$ is positive at room temperature, it becomes negative at around $500 \mathrm{~K}$ and shows a sharp maximum at $T_{c}$, implying that the primary effect dominates beyond this particular temperature. The contributions from the primary and the secondary effects, at different temperatures, can be estimated if the temperature dependence of the elastic, piezoelectric, and the thermal expansion coefficients are known. Unfortunately, at present, no experimental data with regard to the temperature dependence of the piezoelectric, elastic, and thermal coefficients are available in the literature, in order to quantify the primary and the secondary contributions.

\section{G. Ferroelectric properties}

The ferroelectric hysteresis loops for the $\mathrm{KCl}$ modified grain-oriented $(f=0.75)$ ceramic, recorded with the switching field applied in the directions parallel and perpendicular to the pressing axis, are shown in Figs. 14(a) and 14(b). Figure 14(c) shows the hysteresis loop for a randomly oriented additive-free $\mathrm{BiV}$ ceramic sample. It is clear from the figure that the hysteresis loop obtained along the perpendicular direction is nearly rectangular. The coercive field $\left(E_{c}\right)$ and remnant polarization $\left(P_{r}\right)$, computed from the hysteresis loop in each case, are listed in Table II. The $P_{r}$ value is higher along the normal direction, confirming that the polar axis is in the a-b plane. On the other hand, the $E_{c}$ value is higher along the parallel direction, indicating that a large field is required to switch the spontaneous polarization along the $c$-axis. The better hysteresis loops obtained for the $\mathrm{KCl}$-modified samples may be attributed to the lower electrical conductivity associated with these samples. Nevertheless, the influence of the domain shape and size on the ferroelectric hysteresis loop cannot be ruled out. In fact, the higher $E_{c}$ values of the KCl-modified sample

TABLE II. Ferroelectric and pyroelectric properties of KCl-modified grain-oriented ceramic and single crystals of BiV.

\begin{tabular}{|c|c|c|c|}
\hline & $\begin{array}{l}\text { KCl-modified BiV } \\
\text { ceramic }(f=0.75)\end{array}$ & $\begin{array}{l}\text { Randomly oriented } \\
\text { BiV ceramic [20] }\end{array}$ & $\begin{array}{l}\text { Single crystal (along the } \\
\text { polar } a \text { - and } c \text {-axes) [20] }\end{array}$ \\
\hline$P_{r}\left(\mathrm{C} / \mathrm{cm}^{2}\right)$ & $\begin{aligned} P_{r \perp} & =2.06 \times 10^{-8} \\
P_{r \|} & =0.86 \times 10^{-8}\end{aligned}$ & $2.25 \times 10^{-8}$ & $\begin{array}{l}\left(P_{r}\right)_{a}=5.25 \times 10^{-8} \\
\left(P_{r}\right)_{c}=5.7 \times 10^{-9}\end{array}$ \\
\hline$E_{c}(\mathrm{~V} / \mathrm{cm})$ & $\begin{aligned} E_{c \perp} & =1100 \\
E_{c \|} & =2450\end{aligned}$ & 650 & $\begin{aligned}\left(E_{c}\right)_{a} & =500 \\
\left(E_{c}\right)_{c} & =600\end{aligned}$ \\
\hline$p\left(\mu \mathrm{Cm}^{-2} \mathrm{~K}^{-1}\right)$ & $\begin{aligned} p_{\perp} & =500 \\
p_{\|} & =150\end{aligned}$ & 900 & \\
\hline$f_{Q}\left(\times 10^{6} \mathrm{~V} \mathrm{~m}^{-1} \mathrm{~K}^{-1}\right)$ & $\begin{aligned} f_{Q \perp} & =0.61 \\
f_{Q \|} & =0.41\end{aligned}$ & 0.254 & \\
\hline
\end{tabular}




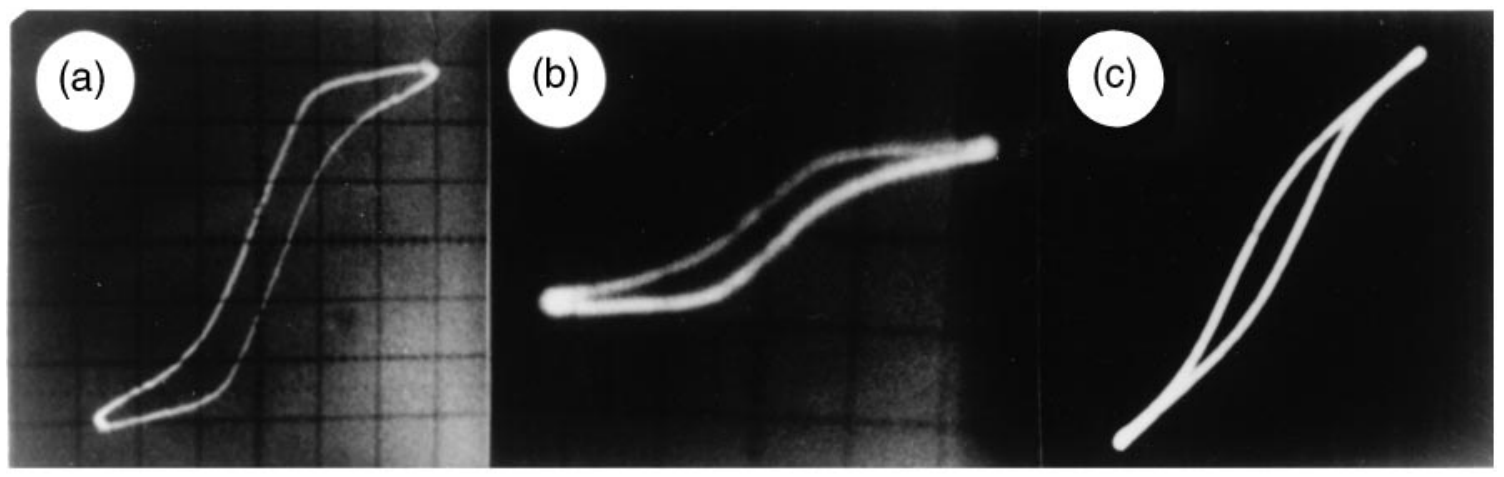

FIG. 14. D-E hysteresis loop recorded with the electric field direction (a) parallel and (b) perpendicular to the pressing axis of the KCl-modified grain-oriented ceramic $(f=0.75)$, and $(c)$ is for the additive-free randomly oriented BiV ceramic.

indicates that the polarization reversal is hindered due to the presence of strongly pinned domain walls, as the defects pin the domain walls and prevent nucleation and growth of domains in the direction favored by an applied electric field. The reduced $P_{r}$ value also indicates that the degree of switchable polarization decreases dramatically in the KCl-modified sample.

\section{CONCLUSIONS}

Grain-oriented ceramics of $\mathrm{BiV}$ are fabricated via simple cold-pressing and liquid-phase aided sintering techniques, by utilizing $\mathrm{KCl}$ as an additive. The high degree of orientation of the grains was established by $\mathrm{x}$-ray powder diffraction and scanning electron microscopic studies. The large anisotropy in the electrical resistivity, dielectric, piezoelectric, pyroelectric, and ferroelectric data obtained for these ceramic samples confirms the grain orientation. The influence of the $\mathrm{KCl}$ amount and the sintering time on the degree of grain orientation was studied. The SEM and EDX analyses indicate that the distribution of $\mathrm{KCl}$ is not uniform and segregates primarily at the grain boundaries forming core-shell-like grain structure. The complex "wavy" domain pattern observed on the $\mathrm{KCl}$-modified $\mathrm{BiV}$ sample indicates the possibility of a strong domain-wall pinning. The combination of core-shell grain structure and the domain-wall pinning has a profound influence on their dielectric-temperature characteristics and the polar properties. Significant anisotropy in the dielectric constant $\left(\epsilon_{r \perp} / \epsilon_{r \|}=1.5\right.$ at $300 \mathrm{~K}$ and 2.76 at $T_{c}$, for the ceramic with $f=0.75$ ) was noticed in these ceramic samples. More importantly, the KCl-modified grain-oriented ceramics exhibit a significant piezoelectric effect, unlike the additive-free randomly oriented ceramics. Further, the ferroelectric hysteresis loops obtained, for the grainoriented samples, by applying the switching field in the direction perpendicular to the pressing axis, is nearly rectangular with higher $P_{r}$ and lower $E_{c}$ values, as compared with those obtained in the parallel direction. These ceramic samples show positive pyroelectric coefficient, indicating that the secondary pyroelectric effect has a substantial contribution to the observed effect. They show pyroelectric figures of merit for quick evaluation of the material, comparable with that of the well-known pyroelectric detector material $\mathrm{LiTaO}_{3}$.

\section{ACKNOWLEDGMENT}

We wish to acknowledge the help rendered by $\mathrm{Mr}$. Sam Philip in conducting the SEM studies.

\section{REFERENCES}

1. A. A. Bush and Yu. N. Venevtsev, Russ. J. Inorg. Chem. 31 (5), 769 (1986).

2. V.G. Osipyan, L.M. Savchenko, V.L. Elbakyan, and P.B. Avakyan, Inorg. Mat. 23, 467 (1987).

3. V. N. Borisov, Yu. M. Poplavko, P. B. Avakyan, and V.G. Osipyan, Sov. Phys. Solid State 30 (5), 904 (1988).

4. K.B.R. Varma, G. N. Subbanna, T.N. Guru Row, and C. N.R. Rao, J. Mater. Res. 5, 2718 (1990).

5. K. V. R. Prasad and K. B. R. Varma, J. Mater. Sci. 30, 6345 (1995).

6. K. V.R. Prasad and K. B. R. Varma, Mater. Chem. Phys. 38, 406 (1994).

7. F. Abraham, M.F. Debreuille Gresse, G. Mairesse, and G. Nowogrocki, Solid State Ionics 28-30, 529 (1988).

8. J.B. Goodenough, A. Manthiram, M. Paranthaman, and Y.S. Zhen, Solid State Ionics 52, 105 (1992).

9. K. Shantha, S. Phillip, and K. B. R. Varma, Mater. Chem. Phys. 48 (48), (1997).

10. K. Shantha and K.B. R. Varma, J. Mater. Chem. 7 (8), 1565 (1997).

11. T. Takenaka and K. Sakata, Jpn. J. Appl. Phys. 19 (1), 31 (1980).

12. H. Igarashi, K. Matsunaga, T. Tanai, and K. Ogazaki, Am. Ceram. Soc. Bull. 57 (9), 815 (1978).

13. K. Sakata, T. Takenaka, and K. Shoji, Ferroelectrics 22, 825 (1978).

14. F. K. Lotgering, J. Inorg. Nucl. Chem. 9, 113 (1959).

15. R. L. Byer and C. B. Roundy, Ferroelectrics 3, 333 (1972).

16. W. D. Kingery, H. K. Bowen, and D. R. Uhlmann, Introduction to Ceramics (John Wiley \& Sons, New York, 1976).

17. C. F. Yang, Jpn. J. Appl. Phys. 36, 3548 (1997).

18. Q. Tan, Z. Xu, J. F. Li, and D. Viehland, J. Appl. Phys. 80, 5866 (1996). 
19. Q. Tan, J. F. Li, and D. Viehland, Philos. Mag. B 76 (1), 59 (1997).

20. K. V. R. Prasad, Ph.D. Thesis, Indian Institute of Science (1994).

21. L. E. Cross, Ferroelectrics 151, 305 (1994).

22. K. Uchino and S. Nimura, Ferroelectrics Lett. 44, 55 (1982).

23. B. Jimenez, J. De Frutos, and C. Alemany, J. Phys. Chem. Solids 48 (10), 877 (1987).
24. W. K. Choo and M. H. Lee, J. Appl. Phys. 53, 7355 (1982).

25. T. R. Armstrong and R. C. Buchanan, J. Am. Ceram. Soc. 73 (5), 1268 (1990).

26. Y. Park and S. A. Song, Mater. Sci. Eng. B47, 28 (1997).

27. G. Mairesse, Fast Ion Transport in Solids, edited by B. Scrosati (Kluwer Academic Publishers, New York, 1993), p. 271. 